\title{
HIGHLIGHTS
}

Chemokines:

http://www.els.net/els/FDA/default.a sp?id=9FF174BB-58D3-4CB59674-7EA4D10BBBA2

SENSORY SYSTEMS

\section{Chemokines inflame the pain}

A point of convergence between neuropathic and inflammatory pain states that might be exploited therapeutically has been identified by $\mathrm{C}$. Abbadie et al. By tracking the nociceptive responses of chemotactic cytokine (chemokine) receptor 2 (CCR2)knockout mice to the induction of inflammation or neuropathy, the team highlighted an important role for Gprotein-coupled chemokine receptors in the processing of chronic pain signals.

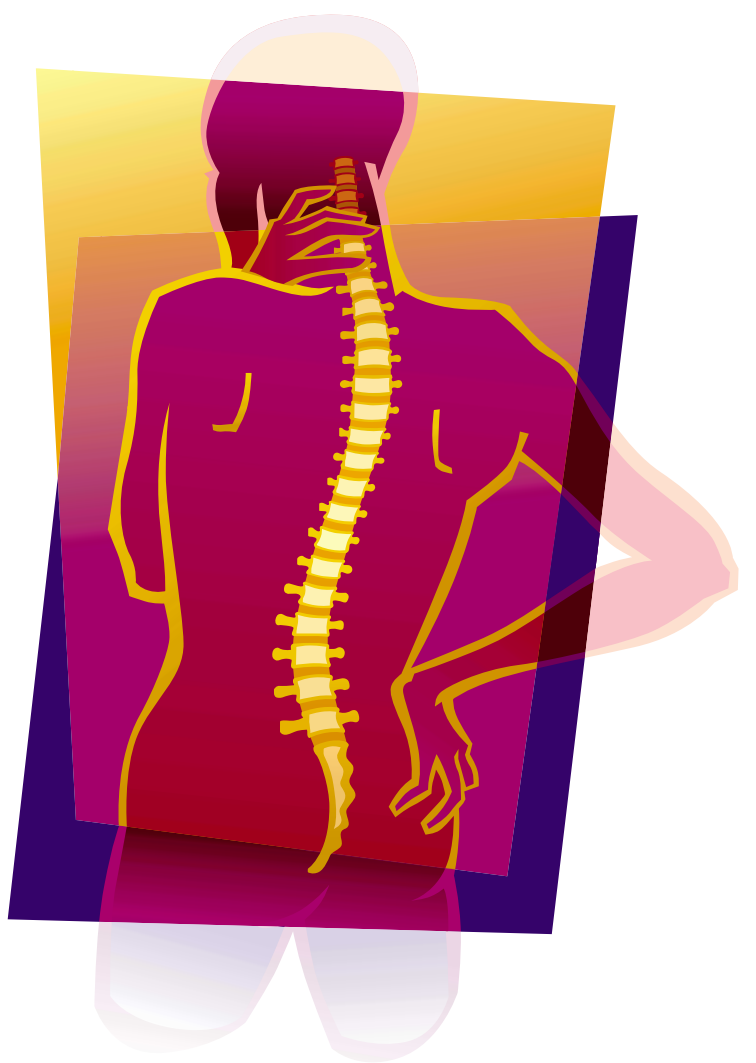

In wild-type mice, mechanical allodynia — a state in which ordinarily non-noxious stimuli cause pain develops after experimental induction of either neuropathy, by partial ligation of the sciatic nerve, or inflammation, by intraplantar injection of Freund's adjuvant. By contrast, mice lacking CCR2 were not hypersensitive to the same stimuli following the same treatments. This result was specific for chronic pain - there were no differences between wild-type and CCR2deficient mice, in response to acutely painful stimuli.

These behavioural data indicate that CCR2 participates in the relay of chronic pain signals, but at which point in the processing pathway does it do so? Abbadie et al. used real-time polymerase chain reaction and immunohistochemistry to address this question. In wild-type mice, mechanical allodynia resulting from nerve damage was accompanied by an increase in the number of CCR2positive monocytes/macrophages, both in the affected nerve and in the dorsal root ganglion. Activated microglia in the spinal cord were also found to express CCR2.

As monocyte chemoattractant protein 1 preferentially binds to CCR2, the authors suggest that the inhibited pain response of mice that lack this receptor might be a consequence of reduced macrophage infiltration at the injured site. This in turn would slow the rate of Wallerian degeneration, the process by which myelin and axonal material are removed from nerves, and which thereby contributes to neuropathic pain. So, targeting chemokine receptors might lead to new treatments for chronic pain syndromes.

Suzanne Farley

(2) References and links

\section{ORIGINAL RESEARCH PAPER Abbadie, C. et}

al. Impaired neuropathic pain responses in mice lacking the chemokine receptor CCR2. Proc. Nat Acad Sci. USA 100, 7947-7952 (2003)

FURTHER READING Tran, P. B. \& Miller, R. J.

Chemokine receptors: signposts to brain development and disease. Nature Rev. Neurosci. 4, 444-455 (2003)

\section{WEB SITE}

Encyclopedia of Life Sciences:

http://www.els.net/

Chemokines 